\title{
La mirada occidental hacia el otro: dos siglos de difíciles encuentros
}

\author{
Álvaro Girón Sierra (*) \\ $\left(^{*}\right)$ Departamento de Historia de la Ciencia de la Institución Milá y Fontanals-CSIC. \\ agiron@imf.csic.es
}

Dynamis

[0211-9536] 2009; 29: 17-28

La expansión europea, que comienza a desarrollarse de manera palpable desde los descubrimientos de los navegantes portugueses a partir del $\mathrm{XV}$, alcanza un impresionante desarrollo en el periodo que va desde mediados del XVIII a la Gran Guerra, entrando en crisis con los procesos de descolonización posteriores a 1945. Es bien sabido que la actividad científica, en la medida que era un instrumento geopolítico de primer nivel, tuvo un papel enormemente relevante en el proceso. A la vez que se abren nuevos mercados y se establecen nuevos equilibrios de poder, mares y continentes son meticulosamente cartografiados, nuevas especies vegetales y animales catalogadas, otros cuerpos y mentes sometidas a la mirada médica. Mientras tanto, en las metrópolis se establecen grandes museos de ciencias naturales, lugares en los que no sólo se impulsa y divulga la investigación científica ${ }^{1}$, sino en los que se celebra la inexorable marcha de los estados-nación europeos; su capacidad de establecer conocimientos y poderes globales. Nuevas disciplinas toman carta de naturaleza en estrecha conexión con este proceso - la Etnología, la Antropología Física - mientras que otras subdisciplinas dentro de la medicina -la medicina tropical, por ejemplo-comienzan su desarrollo. Por otro lado, durante el largo periodo en que la ciencia de laboratorio

1. Este trabajo ha sido redactado dentro del marco del proyecto «La Ciencia europea y su impacto. De el origen a la recepción y desarrollo del darwinismo en España: interpretación, polémicas y aniversario», de referencia HUM 2007-65125-C002-01. Golinski, Jan. Making natural knowledge. Constructivism and the history of science. Cambridge: Cambridge University Press; 1998, p. 95-98. 
todavía no había establecido una primacía indiscutible, la forma más habitual de acreditarse como naturalista era a través del viaje científico. Viajes que, como muestran muy a las claras las biografías de Charles Darwin, Alexander von Humboldt, Thomas Huxley o Piotr Kropotkin, estaban de una manera u otra ligados a los intereses y necesidades de los imperios emergentes.

Como reveló de manera dramática la conquista de México y el Perú, dicha expansión europea llevó a encuentros con otros grupos humanos con desenlaces poco afortunados. Por tanto, el viajero - naturalista o médico- del amplio período que abarca este dossier, no sólo entra en contacto directo con otras culturas extra-europeas, sino que lo hace a partir del conocimiento de una historia anterior $-\mathrm{y}$ muchas veces bien presente- de conflictos. No se puede entender, por ejemplo, la mirada que el joven Darwin desarrolló de los nativos de diferentes tierras de América y el Pacífico, sin tener en cuenta la esclavitud y el fervor abolicionista que heredó de su familia. Es más, es muy posible, como han puesto de manifiesto recientemente Adrian Desmond y James Moore, que la motivación última que impulsó al joven Darwin a elaborar su propia versión del proceso evolutivo - una teoría, recordémoslo, de la descendencia común, que unía inextricablemente en una misma comunidad de sangre no sólo a las distintas razas humanas, sino a éstas con los animales - fuera ofrecer una fundamentación biológica al antiesclavismo, dar un golpe mortal a las emergentes justificaciones científicas de la esclavitud, «(...) a un imperio global del mal que requería una solución global». Pero tampoco se entendería ese fervor abolicionista si no se lo viera como parte integral de una ideología Whig ampliamente compartida por su clase social. Lo cual genera no pocas contradicciones, de manera muy fundamental entre ese fervor humanitarista (que se extendía a todas las criaturas vivientes) y la fábrica malthusiana de la propia teoría de la selección natural que casi de manera inevitable lleva a naturalizar el genocidio y la jerarquía racial:

«El "gran apretujamiento de la población” de Malthus tenía como resultado el conflicto y la conquista, y Darwin comenzó a naturalizar el genocidio en estos términos. Darwin estaba transformando las contingencias de la historia colonial en una ley de la historia natural. Una jerarquía implícita —en la que al hombre blanco se le asignaba el "mejor" intelecto- aseguraba que el colonizador ganaría cuando las culturas entraran en conflicto (...) 
Ligada tan tempranamente a su matriz evolucionista, esta imagen supremacista sería traída posteriormente a colación para justificar políticas de limpieza étnica, por muy aborrecibles que fueran para los propios ideales humanitarios de Darwin» ${ }^{2}$.

Como vemos, no sólo no hay mirada inocente del viajero científico sino que la manifestación pública de dicha mirada puede tener consecuencias. Asumiendo este punto de vista, este dossier ofrece una primera panorámica del papel de científicos y médicos en la construcción de las distintas miradas «occidentales» al «otro», que como es patente no tienen por qué estar exentas de contradicciones. Como no podría ser de otra manera, se tienen muy en cuenta la multiplicidad de contextos que están en su origen, las diferencias en el tiempo y el espacio, sin asumir, por tanto, que exista una sola mirada como tal. Diferencias que pueden ser, por ejemplo, de orden geográfico y político. Thomas Huxley, por ejemplo, es un viajero a las órdenes de un imperio naval, mientras que la experiencia siberiana de Piotr Kropotkin sólo se entiende dentro de las coordenadas de la gran expansión terrestre de los rusos en Asia. Por ello se entendió desde el principio que el dossier debía reflejar esa diversidad geográfica e histórica. La presencia europea en América, Asia y África se desarrolla en circunstancias históricas muy diversas; en no pocas ocasiones la imagen que se proyecta de las culturas y pueblos no europeos depende de circunstancias coyunturales en que la política colonial de cada momento tiene un papel decisivo. Por otro lado, las divisiones del espacio social intervienen de manera no menos importante. La historiografía ha revelado que no era casualidad que un viajero independiente, de escasos recursos, y convencido socialista owenita, como Alfred Russel Wallace, acabara por desarrollar una perspectiva sobre los «primitivos» bien distinta de la de un gentleman de alta clase media como Darwin ${ }^{3}$.

2. "Malthus's "grand crush of population" resulted in conflict and conquest, and Darwin began to naturalize the genocide in these terms (...) Darwin was turning the contingencies of colonial history into a law of natural history. An implicit ranking — with the white man accorded the 'best' intellect - ensured the colonist won when cultures clashed (...) Wedded so early to his evolutionary matrix, this supremacist image would itself be brought to justify later ethnic-cleansing policies, however abhorrent to Darwin's own humanitarian ideals». Desmond, Adrian; Moore, James. Darwin's sacred cause. Race, slavery and the quest for human origins. Londres: Penguin; 2009, p. 108, 148.

3. Moore, James. Wallace's Malthusian moment: the common context revisited. In: Lightman, Bernard, ed. Victorian science in context. Chicago-Londres: Chicago University Press; 1997, p. 
Evidentemente, los viajeros o médicos coloniales tienen unas expectativas previas sobre sus encuentros con otros pueblos y culturas. En esa imagen preconstruida intervienen ideas sobre «salvajes» $\mathrm{y}$ «bárbaros» que remiten a tradiciones ancestrales ${ }^{4}$. Pero también son muy relevantes los relatos de otros viajeros, y, a partir de mediados del XIX, los diversos conceptos de evolución cultural que comenzaban a construir etnólogos y antropólogos (tanto físicos como culturales) ${ }^{5}$. Así, el viajero lleva consigo todo un bagaje científico-cultural en las espaldas que es un elemento mediador ineludible: Kropotkin, por ejemplo, explora Siberia y Manchuria leyendo a Humboldt y tratando de confirmar las teorías darwinianas ${ }^{6}$. Por otra parte, y desde un punto de vista aún más radical, cabe preguntarse si ese discurso alrededor de los otros no es esencial para construir la propia identidad europea a través de la creación de la diferencia, de la distancia entre las normas culturales de unos y otros, cuando no adornadas con diferencias somáticas. Dicho de otra manera, la pregunta es si la creación del barbarismo no es la condición misma de la idea de civilización. En todo caso, esa imagen preconstruida también puede ser modificada o al menos desafiada. Y eso es así porque los «otros» no son entes pasivos plásticamente sometidos a la disciplina de la mirada occidental o a un poder colonial abrumador ${ }^{7}$. De ahí que siempre haya algo de inesperado en el momento del encuentro, cuando el científico pone en juego no ya su imagen preconstruida, sino sus propios sentimientos. Así fue en el caso de Darwin:

«El ambiente claustral de Cambridge podría haber dado a Darwin su anclaje moral, pero él abandonó Inglaterra sin timón. El pudo haber asimilado el ethos antiesclavista de sus hermanas y devorado los tratados de Shrewsbury y Maer, aunque sus áridas enseñanzas resultaran sobre todo patetismo impreso,

290-311; Jones, Greta. Alfred Russel Wallace, Robert Owen and the theory of natural selection. British Journal for the History of Science. 2002; 35: 73-96.

4. Stoczkowski Wiktor. Essai sur la matière première de l'imaginaire anthropologique. Revue de Synthèse. 1992; 113 (4): 439-457.

5. Aunque se puede decir que darwinismo y evolucionismo cultural fueron desarrollos paralelos, su mutua influencia fue destacable. Véase Bowler, Peter J. From «savage» to «primitive». Victorian evolutionism and the interpretation of marginalized peoples. Antiquity. 1992; 66: 721-729.

6. Todes, Daniel P. Darwin without Malthus. The struggle for existence in Russian evolutionary thought. Oxford: Oxford University Press; 1989, p. 123-142; Kropotkin, Piotr. Memoirs of a revolutionist. Nueva York: Dover Publications; 1988, p. 98-99.

7. Sobre esa tensión entre construcción del discurso colonial y resistencia, véase Medina Doménech, Rosa María. La historia de la medicina en el siglo XXI. Una visión poscolonial. Granada: Universidad de Granada; 2005, p. 42-45. 
algo para ser estibado como equipaje ético. Para que el joven Darwin sintiera y comprendiera fue necesario dolor tropical, piernas encadenadas reales y espaldas rotas empapadas por el sudor en los cañaverales de azúcar» ${ }^{8}$.

Y es que, ya no es la propia mirada del científico la que se somete a riesgos insospechados durante el viaje, sino eso que llamamos ciencia.

Cuando el joven Darwin empieza a escribir privadamente sus cuadernos de notas en que se aventura a proponer una teoría evolutiva en los años 1830, ya se están poniendo los fundamentos del moderno racismo científico, siendo desde el principio muy consciente de ello. Sin embargo, todo indica, que a pesar de que algunos naturalistas ya estaban empezando a afirmar la superioridad del europeo mediante el establecimiento de una jerarquía racial de base somática, no se puede decir que en el siglo XVIII el racismo constituyera una visión del mundo como en el XIX ${ }^{9}$. Ello no impide que los relatos de los viajeros estuvieran saturados de prejuicios sobre otras culturas, afirmándose casi siempre la superioridad de la civilización cristiana y occidental. Tal es el caso del manuscrito del Viage a Esmirna, como relata Guillermo Olagüe en el primer artículo de este dossier. El manuscrito, escrito por el cirujano sevillano Pedro María González, pudo ser posible al formar parte de la expedición político-comercial que el Consulado de Cádiz había emprendido fletando en 1796 una fragata cargada de productos americanos en dirección a la ciudad otomana de Esmirna. Este antiguo participante del viaje de Alejandro Malaspina, recibió de los cónsules el encargo de redactar un tratado donde expusiera los medios para facilitar las relaciones comerciales de otros españoles con las ciudades mercantiles otomanas. El texto contiene numerosas consideraciones etnográficas, de las cuales las más originales son las dedicadas a un grupo confesional con el que se podía comunicar con más facilidad: los hebreos sefardíes.

El artículo de Guillermo Olagüe reviste un especial interés por la propia ambigüedad que representaba el imperio otomano para los europeos en esa época. A caballo entre Asia y Europa, los otomanos eran herederos de lo más granado de la cultura occidental - el mundo griego- pero a la

8. "Cloistered Cambridge might have given Darwin his moral anchorage, but he had left England rudderless. He might have imbided his sisters' anti-slavery ethos, devoured Shrewsbury and Maer's tracts, but their dry teachings were so much print pathos, to be stowed as ethical baggage. For the young Darwin to feel as well to understand took tropical pain, real shackled legs and sweat-soaked backs broken in cane fields». Desmond; Moore, n. 2, p. 108.

9. Medina Doménech, n. 7, p. 49 
vez constituían un abigarrado conjunto de grupos étnicos y religiosos que se percibía como especialmente ajeno. Es una tensión cultural que se hace especialmente patente en Esmirna. La estancia de Pedro María González, que se prolongó por dos años, se sitúa dentro las coordenadas de un cambio de actitud general hacia el mundo otomano a lo largo de la Ilustración, desde la curiosidad no exenta de cierta simpatía hacia los turcos, al rechazo generalizado del sistema político otomano a partir de finales del último cuarto del XVIII, posiblemente motivado por la percepción de la debilidad política y militar de la Sublime Puerta. Téngase en cuenta, en todo caso, que esa visión crecientemente prejuiciada de los heterogéneos habitantes del imperio, no iba acompañada de ningún intento de gradación racial de base somática. Los numerosos defectos de las poblaciones locales se debían a razones religiosas - la doctrina de la predestinación-, políticas - el absolutismo- o a la influencia perniciosa del clima.

Pedro María González se centra en una población en la que no encuentra el obstáculo de la barrera idiomática, los judíos sefardíes. Comparte con otros españoles que tuvieron relación con los hebreos otomanos un dualismo entre el acercamiento y el rechazo, en su caso concreto entre una crítica generalizada que sin embargo matiza con su admiración por la capacidad comercial y de hacer negocios de los sefardíes. Es igualmente revelador que González insistiera en crear una distancia casi insalvable entre las costumbres otomanas y españolas, aún cuando otros viajeros percibieran similitudes entre Esmirna y las ciudades de Andalucía. Distancia construida ya sea a partir del rechazo, ya sea bajo el paraguas de la curiosidad, al representarse las costumbres de los osmanlíes como totalmente nuevas.

Sandra Rebok se ocupa de una figura de talla mundial, un viajero y naturalista que se convierte en uno de los vectores fundamentales del cambio desde la ciencia ilustrada a la romántica: Alexander von Humboldt. Un científico que no sólo tuvo una influencia profundísima entre sus coetáneos, sino entre los naturalistas y biólogos de generaciones sucesivas, incluido el joven Darwin, en quien es perceptible no sólo la impronta de la descripción romántica, que asume la subjetividad, sino su débito a la geografía botánica humboldtiana ${ }^{10}$. Rebok adopta un enfoque delibe-

10. El trabajo de Sandra Rebok se enmarca dentro un proyecto de amplio calado encabezado por Miguel Ángel Puig-Samper, que ya ha dado notables resultados. Véase, por ejemplo, Puig-Samper Mulero, Miguel Ángel; Rebok, Sandra. Sentir y medir. Alexander von Humboldt en España. Aranjuez: Doce Calles; 2007. 
radamente holístico, lo cual le lleva a estudiar no tanto la interacción de Humboldt con grupos concretos, sino el complejo retrato que el naturalista prusiano proporcionó de la estructura misma de las sociedades coloniales a las puertas del proceso que convertiría en repúblicas independientes la mayor parte del territorio anteriormente bajo el dominio español. Uno de los valores añadidos fundamentales del artículo es que reposa fundamentalmente sobre documentación procedente de los escritos privados de Humboldt lo cual permite no sólo asomarse a las consideraciones espontáneas que hacía el prusiano sobre el sistema colonial, sino también evitar las nada infrecuentes interpretaciones anacrónicas de las descripciones humboldtianas de las sociedades coloniales. En efecto, las que aparecen en trabajos posteriormente publicados están indudablemente influidas por desarrollos históricos ulteriores, fundamentalmente por los procesos de independencia y creación de las nuevas repúblicas americanas. Además, los diarios de Humboldt proporcionan la única descripción completa de su expedición americana.

Rebok profundiza más allá del rechazo humboldtiano del colonialismo o de la esclavitud, entrando de lleno en la prolija descripción que el naturalista hizo de las distintas sociedades coloniales. En esa descripción se revela un análisis diferenciado de cada sociedad colonial, poniendo un especial acento en las diversas formas de acomodación de las distintas poblaciones (indígenas y esclavas) y los inmigrantes europeos. Mientras que en el Caribe existía una clara separación entre las poblaciones blanca y negra, el sistema social en México, Venezuela, Colombia, Perú y Ecuador era más complejo: se trataba de sociedades de castas en las que factores como la raza, el grado de libertad y el color de la piel eran determinantes. Llama especialmente la atención que Humboldt, aún albergando prejuicios sobre los distintos grupos raciales (hace referencias continuas a la indolencia de los indios), tenía sin embargo un genuino aprecio por el alto grado de desarrollo de las civilizaciones prehispánicas, estableciendo vínculos entre dicho grado y las características idiosincrásicas de cada sociedad colonial. En todo caso, su enfoque no parte de la idea de que existan distinciones fijas y esenciales entre los grupos humanos y razas, sino que esas diferencias se deben a factores ambientales como el clima, o más propiamente culturales o políticos, como el efecto - para el prusiano nefasto-que tiene la acción de los misioneros sobre los indios.

Jerarquía racial y justificación científica de ésta, que sin embargo, ya están plenamente consolidadas en Europa Occidental cuando el Médico 
Mayor Militar Felipe Ovilo Canales (1850-1909) despliega su actividad en Marruecos, dentro de un periodo en el que todo el mapa del mundo se estaba viendo alterado por un reparto colonial en el que a España solo se le acabarán asignando las migajas. Mucho se ha discutido por otro lado, si el racismo científico - en combinación más o menos estrecha con el darwinismo- constituía el discurso dominante a la hora de proporcionar cobertura ideológica a la nueva expansión imperial y al colonialismo. Paul Crook, por ejemplo, afirma que la gran mayoría de los argumentos a favor de la expansión colonial británica hacía muy poca referencia a las teorías de la evolución humana. Fueron más bien argumentos de orden estratégico, patriótico y económico, y los debates eran tratados en los términos de moralidad y teodiceas tradicionales ${ }^{11}$. En todo caso, y por discutible que pueda ser esta afirmación, lo cierto es que el discurso colonial no es uniforme. Puede manifestar matices distintos en cada país, y muy bien podría ser que no obedezca a un único patrón o incluso establezca diversas afinidades electivas con distintas teorías biológicas. En el caso francés, Jean Marc Bernardini habla de una fraseología darwiniana de la concurrencia, pero «atemperada por un deber de las razas superiores frente a las razas inferiores» ${ }^{12}$. Influencia darwiniana, sin embargo, que algunos autores si ven más evidente en Alemania, sobre todo en los años anteriores a la Gran Guerra ${ }^{13}$.

Dejando al margen los grandes debates, lo cierto es que resulta necesario tocar tierra, saber mucho más sobre la ideología y mentalidad de los que estaban embarcados en la aventura colonial de manera efectiva. Y más teniendo en cuenta la no uniformidad del discurso colonial, tal como revela de manera extraordinariamente convincente el artículo de Javier Martínez Antonio sobre el citado Felipe Ovilo. Lejos de los discursos de franca superioridad de tipo colonialista o civilizador más visible en otros países, la aproximación de Ovilo se movía dentro de los límites del movimiento africanista de su época, permeado de regeneracionismo, y que operaba

11. Crook, Paul. Historical monkey business: The myth of a Darwinized British imperial discourse. History. 1999; 84 (276): 633-657.

12. Bernardini, Jean-Marc. Le darwinisme social en France (1859-1918). París: CNRS Éditions; 1997, p. $44,401$.

13. Evans, Richard. J. In search of German social Darwinism. The history and the historiography of a concept. In: Berg, Manfred; Crock, Geoffrey, eds. Medicine and modernity: Public health and medical care in 19th and 20th century Germany. Cambridge: Cambridge University Press; 1997, p. 55-79. 
bajo el convencimiento de que no era posible desplegar una acción estrictamente colonialista en Marruecos. Para el doctor el objetivo de España habría de ser colaborar en las reformas del estado marroquí, y no por la fuerza militar, sino en nombre de la superioridad moral: su propia labor sanitaria se ajustó a los presupuestos generales de intervención amistosa o regeneración. Por otra parte, aunque la raza ocupa un lugar importante en las reflexiones de Ovilo - en las que insiste en la distancia sociocultural de la realidad marroquí con respecto a sus términos de comparación españoles/europeos- su discurso racial careció de una base científica comparable a sus reflexiones sobre las epidemias o la higiene pública. Es más, él percibía una fuerte proximidad racial entre España y Marruecos, con lo que la superioridad racial de los españoles sobre los marroquíes resultaba bastante relativa, no existiendo en la obra de Ovilo un estereotipo unitario del marroquí. La raíz de su discurso racial era histórica y moral, llegándose a columbrarse una convergencia hispano-marroquí - una misma raza- aunque no en pie de igualdad.

Después de la Primera Guerra Mundial, aunque la retórica racial ocupara todavía un lugar principal en el discurso público y, sobre todo, en la política, algunos de los apoyos más sólidos del racismo científico empiezan a cuartearse, muy singularmente el evolucionismo cultural en antropología. No parece que sea éste el impulso que mueve al patólogo e higienista alemán Max Kuczynski-Godard (1890-1967) a rechazar los conceptos de raza y constitución, sino aspectos fundamentales que concernían a la aproximación ética y epistemológica que desarrolló con respecto a la Medicina, como afirma Michael Knipper en el artículo que pone fin a este dossier. Dicho de otra forma, tanto la mirada que desarrolló el médico alemán de las poblaciones nativas con las que estuvo en contacto en dos continentes, como su propia práctica, tienen no poco que ver con su particular respuesta de carácter fundamentalmente ético a lo que él percibía como profunda crisis disciplinar. Kuczynski emprendió importantes expediciones de carácter médico en los años 1920 en Siberia, Mongolia y China, actividad a la que en cierta manera siguió dando continuidad en la Amazonia peruana entre 1938 y 1944, donde se había instalado, entre otras razones, para permanecer al abrigo de la persecución nazi contra los judíos.

Su interés por las poblaciones nativas de esos variados entornos geográficos radicaba en realidad en una crítica epistemológica de la medicina científica que se había consolidado desde mitad del siglo XIX. El médico 
alemán, aunque no participara de manera directa, se vio envuelto en la polémica sobre la crisis de la medicina en la Alemania de Weimar. Las críticas a una medicina que sólo parecía preocuparse de la dimensión material de la enfermedad en detrimento del hombre enfermo, el desacuerdo con la idea de tomar a las ciencias naturales como única instancia para juzgar el valor de los razonamientos médicos, el énfasis en la necesidad de integrar la dimensión psicológica en la medicina clínica, y el disgusto, en fin, ante la fragmentación de la medicina moderna en un creciente número de disciplinas especializadas, se convierten en moneda corriente. La respuesta de Kuczynski fue extender la investigación empírica más allá de los confines del laboratorio encontrando en la patología histórica y geográfica del XIX el marco teórico específico para incluir factores externos a su concepción: clima, condiciones físicas del ambiente, aspectos sociales, económicos y culturales. La consecuencia epistemológica es integrar las dimensiones sociales, económicas y culturales en la teoría de la enfermedad.

Ahora bien, lo que ilumina de manera más cabal según Knipper el particular enfoque de Kuczynski, que le lleva en la práctica a una asombrosa variabilidad metodológica, son las valoraciones éticas subyacentes. Por un lado, nos encontramos con la percepción del riesgo de perder de vista los valores principales de la medicina ante la fragmentación de la visión etiológica moderna. Dicho de otra forma, le preocupa el hecho de que la medicina sea cada vez más entendida como el estudio de la «enfermedad en sí», en lugar de preocuparse de los enfermos. Pero aún es más visible su compromiso con el humanismo ilustrado y sus valores: la igualdad entre los seres humanos, la confianza en su «educabilidad» y el uso consciente de la razón. Es lo que explica su rechazo de planteamientos discriminatorios fundados en el racismo científico.

Que este dossier finalice con el antirracista e ilustrado Kuczynski, no quiere decir que se pretenda avalar implícitamente una supuesta narrativa progresiva desde los oscuros excesos del racismo científico del XIX hasta la celebración apologética de una genética humana contemporánea, que vendría a dar un golpe mortal al propio concepto de raza. Algunos podrán ver las desafortunadas afirmaciones del Nobel James Watson sobre la inteligencia de los negros como un accidente menor, algo que tiene más que ver con sus propios prejuicios que con la verdadera ciencia. En cierta manera, la propia torpeza de las declaraciones de Watson hacían más viable la operación de distinguir quirúrgicamente los prejuicios del científico 
individual, del supuesto venero de ciencia pura que constituye la genética humana. El científico puede ser problemático y discriminatorio, la Ciencia con mayúsculas no.

A la luz de todo ello, se puede entender mucho mejor cómo, a grandes rasgos, los grandes fastos del doble centenario de otro viajero - Charles Darwin- se están convirtiendo en eventos controlados, en los que décadas de exhaustiva investigación no ya sólo sobre el origen y carácter de sus teorías, sino sobre el naturalista británico de carne y hueso, aparecen extrañamente ausentes. No debe de extrañar: nadie va a invitar a los historiadores aguafiestas, o más exactamente a los más aguafiestas de entre los historiadores, al gran festival legitimatorio de una variedad creciente de disciplinas, cuando no de la ciencia misma. Profesionales de los más variados campos, cuyo conocimiento de la obra escrita de Darwin es más que dudoso, propalan en los medios públicos una rancia imagen del genio aislado que entra en una inadvertida contradicción con la no menos habitual del paciente y modesto observador. Los más sutiles establecen una clínica distinción entre los excesos del santo patrón (propios de su época, dicen), y lo que se hace aparecer como incontaminada teoría científica: la selección natural. Todo ello nada tiene que ver, evidentemente, con el complejo retrato de Darwin que emerge de una de las más y mejor documentadas biografías de la historia de la ciencia: se puede decir con cierta justicia que el doble centenario se está convirtiendo en el segundo secuestro de Darwin, aunque esta vez no haya cuerpo que convenientemente enterrar en Westminster Abbey ${ }^{14}$. Quizás lo más descorazonador de esta operación de rescate de Darwin para la ciencia «neutral», es que se está hurtando aquello que hace más interesante a Darwin para el público contemporáneo, aunque le convierta en una figura mucho más vulnerable. Muy singularmente, como decía hace casi tres décadas John C. Greene, la lucha por reconciliar la convicción darwiniana de que la competencia entre individuos, tribus, naciones, y razas era esencial para el progreso de la humanidad, con el igualmente fuerte sentimiento de las obligaciones de «la humanidad ilustrada» hacia pueblos situados por debajo de los

14. La voluntad inicial del propio Darwin y de su familia no era precisamente ser enterrado en Westminster Abbey. Este hecho fue el resultado de un elaborado plan en el que jugó un papel decisivo el grupo originario de «darwinistas». Véase Moore, James. R. Charles Darwin lies in Westminster Abbey. Biological Journal of the Linnaean Society. 1982; 17: 97-113. 
occidentales en la escala evolutiva ${ }^{15}$. Una hiriente contradicción que muy bien podría estimular la reflexión, o al menos iluminar las muy numerosas ambigüedades de un mundo en apariencia postcolonial.

15. Greene, John C. Essays in the History of Evolutionary Ideas. Berkeley: University of California Press; 1981, p. 114. 